Natália Ramos ${ }^{1}$

Aline Domingues Chaves Aita ${ }^{2}$

Luciana Pillon Siqueira ${ }^{3}$

Fabiano Sbicigo Aita ${ }^{4}$

\section{O uso de emissóes otoacústicas como ferramenta auxiliar no diagnóstico de efeitos da exposição ao ruído*}

\author{
Using otoacoustic emissions as an auxiliary tool for the diagnosis
} of noise exposure effects

\begin{abstract}
${ }^{1}$ Acadêmica do Curso de Graduação em Fonoaudiologia da Faculdade Fátima, Caxias do Sul, RS, Brasil.

2 Fonoaudióloga, Doutora em Distúrbios da Comunicação Humana - Campo Fonoaudiológico. Docente do Curso de Bacharelado em Fonoaudiologia da Faculdade Fátima, Caxias do Sul, RS, Brasil.

3 Fonoaudióloga, Mestre em Ciências dos Distúrbios da Comunicação Humana. Docente do Curso de Bacharelado em Fonoaudiologia da Faculdade Fátima, Caxias do Sul, RS, Brasil

${ }^{4}$ Otorrinolaringologista, Docente do Curso de Bacharelado em Fonoaudiologia da Faculdade Fátima, Caxias do Sul, RS, Brasil
\end{abstract}

*Baseado no trabalho de Natália Ramos desenvolvido para a conclusão do curso de Graduação em Fonoaudiologia da Faculdade Fátima, Caxias do Sul, RS, em 2009

Contato:

Natália Ramos

Rua Duque de Caxias, 2181/301, Bairro Madureira. Caxias do Sul/RS.

CEP: $95020-200$

E-mail:

taia.ramos@yahoo.com.br

\section{Resumo}

A exposição a níveis elevados de ruído é causa de perda auditiva sensorioneural, a qual poderia ser diagnosticada mais precocemente. O objetivo do estudo, realizado em 2009, foi investigar respostas eletroacústicas para as Emissões Otoacústicas Evocadas por Estímulo Transiente (EOAT) e as Emissões Otoacústicas Evocadas Produto de Distorção (EOAPD) em trabalhadores expostos a níveis de ruído iguais ou superiores a 85dB(A). Meatoscopia, imitânciometria, EOAT e EOAPD foram realizados em 270 trabalhadores metalúrgicos do sexo masculino, entre 18 a 30 anos, divididos em GI (160 trabalhadores com 1 a 5 anos de exposição) e GII (110 trabalhadores com exposição maior que 5 anos). Entre as EOAT do GI, 82\% passaram e 5,62\% falharam bilateralmente, já no GII, 80\% passaram e 7,3\% falharam bilateralmente. Entre as EOAPD no GI, 96,9\% passaram e 0,6\% falhou bilateralmente. No GII, 97,3\% passaram e 0,9\% falhou bilateralmente. Diferenças foram significantes para EOAPD entre os grupos, em 4 e $5 \mathrm{kHz}$. Os resultados obtidos mostraram que as respostas para EOAT estiveram ausentes em maior número quando comparadas aos registros das EOAPD, mesmo quando os limiares auditivos obtidos na Audiometria Tonal Liminar estavam normais. Assim, a EOAT é uma importante ferramenta para a detecção precoce de alterações na fisiologia coclear decorrente da exposição ocupacional ao ruído, permitindo que medidas preventivas sejam adotadas mais precocemente pelas empresas.

Palavras-chave: audição; trabalhadores; emissões otoacústicas.

\section{Abstract}

Exposure to high levels of noise cause sensorineural hearing loss that could be detected early. This study, conducted in 2009, investigated electroacoustic responses for Transient Evoked Otoacoustic Emission (TOAE) and for Distortion Product Otoacoustic Emission (DPOAE) in workers exposed to noise levels equal to or above $85 \mathrm{~dB}(\mathrm{~A})$. Meatoscopy, immittance measures, TOAE and DPOAE were performed in 270 male metallurgy workers between 18 and 30 years. Workers were assigned to GI - 160 workers exposed for one to five years, or GII - 110 workers exposed for more than five years. Concerning TOAE, in GI, $82 \%$ passed and $5.62 \%$ failed bilaterally; in GII, $80 \%$ passed and 7.3\% failed bilaterally. For DPOAE, in GI 96.9\% passed and 0.6\% failed bilaterally; in GII, 97.3\% passed and 0.9\% failed bilaterally. There were statistically significant differences between the groups for $D P O A E$ in $4 \mathrm{kHz}$ and $5 \mathrm{kHz}$. Results showed that responses to TOAE were absent in a greater number than those to DPOAE in this group, even when hearing thresholds obtained in pure tone audiometry were normal. Thus, TOAE is an important tool to detect disorders in cochlear physiology due to occupational exposure to noise, and can be useful for implementing earlier measures of protection.

Keywords: hearing; workers; otoacoustic emissions. 


\section{Introdução}

O ruído é um agente físico que está presente no cotidiano dos indivíduos e, principalmente, nos ambientes de trabalho. O surgimento de máquinas, que ficaram cada vez maiores e mais ruidosas, contribuiu para agravar o problema do ruído (FIORINI, 2000). A exposição ao ruído, em níveis elevados de pressão sonora, é uma das mais importantes causas de perda auditiva sensorioneural em nossa sociedade nos dias atuais, sendo possível sua prevenção (OLIVEIRA, 1999; MARQUES, 2003).

A mudança permanente do limiar auditivo ocasionada por exposição a níveis elevados de pressão sonora é denominada Perda Auditiva Induzida por Níveis de Pressão Sonora Elevados (Painpse). A Painpse é uma doença ocupacional de alta prevalência nos países industrializados, destacando-se como um dos agravos à saúde do trabalhador mais prevalentes nas indústrias brasileiras (ALMEIDA et al., 2000).

A investigação das exposições a altos níveis de ruído e suas consequências à audição tem sido uma preocupação constante, envolvendo crescentes estudos para melhor entender e delimitar a ocorrência da Painpse (MARQUES, 2003).

De acordo com Costa (1988), a Audiometria Tonal Liminar (ATL) é o método universalmente adotado e o único sugerido pela Portaria $\mathrm{n}^{\mathrm{o}} 19$ do Ministério do Trabalho e Emprego (BRASIL, 1998). Entretanto, conforme destacado por Marques (2003), não é o melhor meio de avaliação dos distúrbios produzidos pelo ruído, pois é um teste passível de erros, visto depender diretamente das respostas do sujeito sob avaliação (Fiorini, 2000). Outra desvantagem da utilização da audiometria na detecção precoce da Painpse, citada por Oliveira (1999), é a sua baixa sensibilidade para detectar as sutis modificações cocleares que ocorrem antes que a perda auditiva se manifeste no audiograma. Sliwinska-Kowalska (1998) destacou que os programas de conservação auditiva ainda necessitam de outros testes, além da audiometria, que possam identificar os danos auditivos decorrentes da exposição a ruído.

Um complemento à ATL para triar e monitorar possíveis modificações cocleares precoces seria a Análise de Emissões Otoacústicas (EOA). Tal exame é um procedimento rápido, objetivo, não invasivo e capaz de detectar modificações cocleares mínimas (OLIVEIRA, 1999). As EOA são sons que decorrem de uma atividade interna coclear e que são encontrados no Meato Acústico Externo, gerados pela atividade fisiológica dentro da cóclea (SOUSA et al., 2008; MARQUES; COSTA, 2006).

O decréscimo da acuidade auditiva e da sele tividade de frequências provavelmente advém de uma redução da motilidade das Células Ciliadas
Externas (CCEs), acarretando, assim, mudanças nas propriedades das EOA (GATTAZ, 2001).

Suas modalidades mais utilizadas clinicamente são: as Emissões Otoacústicas Evocadas por Estimulo Transiente (EOAT), em que a cóclea é estimulada globalmente, representando o desempenho deste órgão como um todo; e as Emissões Otoacústicas Evocadas - Produto de Distorção (EOAPD), em que é possível verificar o desempenho coclear em frequências específicas (KEMP, 2002).

O presente estudo teve como objetivo investigar as respostas eletroacústicas para as EOAT e para as EOAPD em trabalhadores expostos a ruído.

\section{Método}

A presente pesquisa foi aprovada pelo Comitê de Ética da Faculdade Nossa Senhora de Fátima sob o número 001/08. Trata-se de um estudo transversal, realizado entre fevereiro e novembro de 2009, no qual foram utilizadas as medidas eletroacústicas obtidas em indivíduos expostos ocupacionalmente ao ruído.

Participaram como sujeitos da pesquisa funcionários de uma empresa do ramo metalúrgico de Caxias do Sul, RS, expostos a níveis de ruído iguais ou superiores a $85 \mathrm{~dB}(\mathrm{~A})$. A medição do nível de ruído é realizada anualmente em cada setor pelo Programa de Prevenção de Riscos Ambientais (PPRA) da empresa. Dessa população, foram selecionados os sujeitos do sexo masculino, com idade entre 18 e 30 anos, com tempo mínimo de um ano de exposição ocupacional ao ruído e que apresentaram limiares tonais dentro de limites aceitáveis na Audiometria Tonal Liminar (ATL), conforme Portaria n⿳o 19 do Ministério do Trabalho e Emprego (BRASIL, 1998) - até 25 dBNA em todas as frequências - com configuração plana, na avaliação audiológica periódica realizada pela empresa nos últimos seis meses.

Para a seleção do grupo, estimou-se, através do pacote estatístico Epi Info versão 6.0, uma amostra de 273 sujeitos, considerando uma margem de erro de 3,5\% e nível de significância de 95\%. Para seleção dos sujeitos da pesquisa, foi utilizado um processo de amostragem sistemática.

Após a seleção dos 273 sujeitos, o registro das EOAT e EOAPD foram realizados no Centro de Saúde da empresa, em uma sala com baixo nível de ruído. Todos os indivíduos estavam em repouso acústico de no mínimo 14 horas para realização da bateria de exames.

Após a obtenção do Termo de Consentimento Livre e Esclarecido, realizou-se a investigação audiológica, constituída da inspeção visual do meato acústico externo e de Medidas de Imitância Acústica com o equipamento portátil e automático MT-10 da marca Interacoustics (Interacoustics - Dinamarca). Para a 
comprovação da normalidade do sistema tímpano-ossicular, foram incluídos na amostra somente os indivíduos que apresentaram curva timpanométrica tipo A, ou seja, um pico de máxima compliância ao redor da pressão atmosférica de zero decaPascal (JERGER, 1970). Assim, foram excluídos da amostra 3 sujeitos por apresentarem alterações na curva timpanométrica.

Os resultados obtidos neste estudo constituem-se da avaliação eletroacústica de 270 sujeitos. Para isso, os sujeitos foram divididos em dois grupos a partir do tempo total de exposição ocupacional ao ruído e os resultados foram analisados em função destes grupos: Grupo I (GI): 160 sujeitos (59,3\%) com exposição ocupacional ao ruído entre um e cinco anos; Grupo II (GII): 110 sujeitos (40,7\%) com exposição ocupacional ao ruído por mais de cinco anos, com limite de 15 anos. Em ambos os grupos, foi considerada toda a vida laboral e a exposição pregressa ao ruído.

As medidas eletroacústicas utilizadas para análise dos resultados deste estudo foram obtidas através dos registros das EOAT e EOAPD com o equipamento da marca MAICO, modelo ERO-SCAN ${ }^{\mathrm{TM}}$ (EOA Test System, Maico Diagnostics - Berlin/Alemanhã).

Os parâmetros utilizados no registro das EOAT foram: estímulo do tipo click na intensidade de 83 dBNPS nas bandas de frequências de $1,5 \mathrm{kHz}, 2 \mathrm{kHz}$, $2,5 \mathrm{kHz}, 3 \mathrm{kHz}, 3,5 \mathrm{kHz}$ e $4 \mathrm{kHz}$. No registro das EOAPD, utilizaram dois tons puros simultâneos com diferentes frequências nas intensidades P1 $=65 \mathrm{dBNPS}$ e $\mathrm{P} 2=55 \mathrm{dBNPS}$, nas frequências de $1,5 \mathrm{kHz}, 2 \mathrm{kHz}$ $3 \mathrm{kHz}, 4 \mathrm{kHz}, 6 \mathrm{kHz}$.
Para análise das respostas, tanto para EOAT, quanto EOAPD, usou-se como critério de passa/falha o registro de respostas em pelo menos três frequências, com amplitude das emissões medidas em dB NPS de, pelo menos, $6 \mathrm{~dB}$ acima do ruído de fundo em cada frequência estudada. Além disso, foram analisadas as amplitudes das EOAPD.

Os dados foram tabulados e analisados no pacote estatístico SPSS versão 15.0. Para responder aos objetivos do estudo, foram realizadas análises uni e bivariadas. O nível de significância estabelecido foi de 0,05.

\section{Resultados}

Com relação às respostas obtidas para as EOAT e EOAPD, inicialmente, através do cálculo de frequência simples, analisamos a presença e/ou ausência de resposta em cada um dos protocolos utilizados em função da variável tempo de exposição ocupacional ao ruído.

Assim, na Tabela 1, apresentamos as respostas (passa/falha) obtidas para as EOAT e EOAPD em função da variável orelha para cada um dos grupos estudados.

As respostas obtidas para EOAPD foram analisadas em função da amplitude de resposta por frequência, por orelha e por grupo (Tabela 2). Observa-se que, na orelha direita (OD), houve diferença estatisticamente significante na banda de frequência de 5 $\mathrm{kHz}$, enquanto que, na orelha esquerda (OE), a diferença estatisticamente significante ocorreu na banda de frequência de $4 \mathrm{kHz}$.

Tabela 1 Respostas para EOAT e EOAPD em função da orelha e da exposição ocupacional ao ruído entre trabalhadores metalúrgicos de Caxias do Sul, Rio Grande do Sul, 2009 (N = 270)

\begin{tabular}{|c|c|c|c|c|c|}
\hline \multirow{2}{*}{ Variáveis } & \multicolumn{2}{|c|}{ Grupo I } & \multicolumn{2}{|c|}{ Grupo II } & \multirow{2}{*}{ p-valor } \\
\hline & $n$ & $\%$ & $n$ & $\%$ & \\
\hline \multicolumn{6}{|c|}{ EOAPD - OD } \\
\hline Passa & 157 & 59,0 & 109 & 41,0 & \multirow{2}{*}{$0,46(1)$} \\
\hline Falha & 3 & 75,0 & 1 & 25,0 & \\
\hline \multicolumn{6}{|c|}{ EOAPD - OE } \\
\hline Passa & 157 & 59,5 & 107 & 40,5 & \multirow{2}{*}{$0,47(1)$} \\
\hline Falha & 3 & 50,0 & 3 & 50,0 & \\
\hline \multicolumn{6}{|c|}{ EOAT - OD } \\
\hline Passa & 145 & 59,7 & 98 & 40,3 & \multirow{2}{*}{$0,68(2)$} \\
\hline Falha & 15 & 55,6 & 12 & 44,4 & \\
\hline \multicolumn{6}{|c|}{ EOAT - OE } \\
\hline Passa & 138 & 60,0 & 92 & 40,0 & \multirow{2}{*}{$0,55(2)$} \\
\hline Falha & 22 & 55,0 & 18 & 45,0 & \\
\hline
\end{tabular}

(1) Teste Qui-Quadrado de Fischer (frequência esperada < 5).

(2) Teste Qui-Quadrado de Pearson (frequência esperada > 5). 
Tabela 2 Amplitude das EOAPD** por frequência para cada orelha e tempo de exposição ocupacional a ruído entre trabalhadores metalúrgicos de Caxias do Sul, Rio Grande do Sul, 2009 (N = 270)

\begin{tabular}{|c|c|c|c|c|c|}
\hline \multirow[t]{2}{*}{ Frequência $(\mathrm{kHz})$} & \multicolumn{2}{|c|}{ Grupo I } & \multicolumn{2}{|c|}{ Grupo II } & \multirow[t]{2}{*}{ Valorde $p$} \\
\hline & Média & $D P$ & Média & $D P$ & \\
\hline \multicolumn{6}{|l|}{ Orelha Direita } \\
\hline 1,5 & 12,93 & 7,27 & 13,96 & 6,71 & 0,21 \\
\hline 2,0 & 16,27 & 6,93 & 15,51 & 8,39 & 0,71 \\
\hline 3,0 & 18,84 & 7,27 & 19,22 & 5,86 & 0,88 \\
\hline 4,0 & 18,54 & 6,58 & 17,60 & 6,23 & 0,05 \\
\hline 5,0 & 16,63 & 7,71 & 14,85 & 7,22 & $0,02^{*}$ \\
\hline 6,0 & 15,63 & 8,78 & 14,25 & 8,14 & 0,15 \\
\hline \multicolumn{6}{|l|}{ Orelha Esquerda } \\
\hline 1,5 & 11,93 & 7,09 & 12,52 & 6,99 & 0,41 \\
\hline 2,0 & 14,69 & 6,92 & 15,18 & 7,89 & 0,46 \\
\hline 3,0 & 18,20 & 7,87 & 17,53 & 6,85 & 0,26 \\
\hline 4,0 & 18,65 & 6,31 & 17,35 & 6,28 & $0,02^{*}$ \\
\hline 5,0 & 16,83 & 7,90 & 15,24 & 7,63 & 0,05 \\
\hline 6,0 & 15,81 & 9,14 & 14,14 & 7,91 & 0,12 \\
\hline \multicolumn{6}{|c|}{ Grupo I - exposição entre 1 a 5 anos (n = 160); Grupo II - exposição maior que 5 anos (n = 110) } \\
\hline \multicolumn{6}{|c|}{ DP = desvio padrão. } \\
\hline * Significante; Teste K & Wallis & & & & \\
\hline
\end{tabular}

\section{Discussão}

Com relação ao critério passa/falha em função da variável orelha para cada um dos grupos estudados, apresentado na Tabela 1, observou-se que não houve diferença estatisticamente significante entre os grupos, porém, nota-se que há maior número de falhas nas EOAT, o que poderia sugerir uma alteração leve no funcionamento coclear dos sujeitos investigados, uma vez que todos apresentavam audição dentro dos padrões de normalidade à ATL.

Fiorini (2000) realizou um estudo em que foram pesquisados os registros de EOA de dois grupos de indivíduos de uma indústria têxtil paulista com limiares audiométricos normais: 80 expostos durante toda a jornada de oito horas diárias de trabalho, a níveis médios de 85 a 105 dBA; e 80 não expostos ao ruído ocupacional. O estudo compreendeu tanto o registro das EOAPD, quanto das EOAT. Em relação às EOAT, a autora encontrou ausência de respostas em $48(60 \%)$ sujeitos na OD e $46(57,5 \%)$ na OE no grupo exposto ao ruído ocupacional, enquanto que para as EOAPD, também no grupo exposto a ruído, $13(27,7 \%)$ tiveram ausência de respostas na OD e 9 $(19,1 \%)$ na OE.

Ao comparar os resultados obtidos no presente estudo com os apresentados por Fiorini (2000), uma menor proporção de sujeitos da nossa amostra apresentou resultados alterados para as duas medidas eletroacústicas empregadas. Provavelmente, tal diferença se deva aos critérios empregados para a classificação das respostas obtidas para as EOA em passa/ falha. Porém, observou-se também que, no estudo de Fiorini (2000), o registro das EOAT mostrou-se mais sensível à detecção precoce de lesões nas células ciliadas externas em decorrência da exposição ocupacional ao ruído.

Com relação a análise das amplitudes das EOAPD, na qual foram comparadas as variáveis do tempo de exposição ocupacional ao ruído, ou seja, GI com GII, demonstradas na Tabela 2, verificou-se diferença estatisticamente significante na banda de frequências $5 \mathrm{kHz}$ na OD e na banda de frequência de $4 \mathrm{kHz}$ na OE quando comparamos os dois grupos estudados. Vale destacar que ambas são faixas de frequências agudas e que geralmente são as primeiras a apresentarem alterações auditivas associadas à exposição ao ruído excessivo.

Os autores Atchariyasathian, Chayarpham e Saekhow (2008) realizaram um estudo com o objetivo de avaliar a Painpse através das EOAPD em três grupos de trabalhadores, divididos conforme os resultados audiológicos e o tempo de exposição a ruído (tempo médio de exposição de 15,2 anos): Grupo 1 - grupo controle (audição normal e sem exposição ocupacional ao ruído); Grupo 2 - limiares audiométricos normais com exposição a ruído; e Grupo 3 limiares audiométricos anormais caracterizados por Painpse com exposição a ruído. Como resultado, os 
autores encontraram uma diferença estatisticamente significante entre o grupo controle e os sujeitos com exposição a ruído (grupos 2 e 3). Além disso, encontraram diferença estatisticamente significante entre o grupo 1 e o grupo 2 em todas as frequências estudadas ( $1 \mathrm{kHz}, 1,4 \mathrm{kHz}, 2 \mathrm{kHz}, 2,8 \mathrm{kHz}, 4 \mathrm{kHz}$ e $6 \mathrm{kHz})$.

No presente estudo, verificamos diferença estatisticamente significante para a amplitude das EOAPD somente nas frequências agudas $-5 \mathrm{kHz}$ na OD e 4 kHz na OE - (Tabela 2), discordando parcialmente dos achados descritos no parágrafo acima e dos achados descritos no estudo de Salazar et al. (2003) que, ao compararem as respostas obtidas para as EOAPD em dois grupos, um exposto ao ruído ocupacional [85 a $105 \mathrm{~dB}(\mathrm{~A})$ ] e outro não exposto, não encontraram diferença estatisticamente significante na comparação das respostas obtidas para os grupos constituídos.

Moran e Fiorini (2003) estudaram os registros de EOAPD em 89 sujeitos expostos a ruído [85 a 105 $\mathrm{dB}(\mathrm{A})]$ que apresentaram perdas auditivas neurossensoriais. As autoras avaliaram as amplitudes do teste de EOAPD em quatro intervalos de tempo de exposição ocupacional ao ruído (0,6-8, 8,1-16, 16,1-24, 24,132 anos) em 135 orelhas. Observaram que, quanto maior o tempo de exposição, menor a amplitude das respostas, principalmente nas altas frequências.

Assim como no estudo descrito acima, pôde-se observar no presente grupo que, quanto maior o tempo de exposição ocupacional ao ruído, menores foram as amplitudes das respostas obtidas para as EOAPD, principalmente nas frequências agudas, sendo que, em $4 \mathrm{kHz}$ (OE) e $5 \mathrm{kHz}$ (OD), tal diferença foi estatisticamente significante quando comparamos as respostas obtidas para esse grupo (GII) com as obtidas para o grupo com menor tempo de exposição ocupacional ao ruído (GI), concordando, portanto, com a afirmação de Moran e Fiorini (2003) quanto à influência do tempo de exposição para a redução da amplitude das EOAPD.

Korres et al. (2009) realizaram uma pesquisa com o objetivo de avaliar a Painpse em um grupo de trabalhadores industriais através do registro das EOAPD. Foram incluídas na pesquisa 210 orelhas expostas ao ruído ocupacional comparadas com os resultados de 68 orelhas normais, pareados em sexo e idade. Os autores observaram que uma alta porcentagem das orelhas testadas tinham redução significante na amplitude das EOAPD obtidas para as frequências entre $3 \mathrm{kHz}$ e $6 \mathrm{kHz}$, sendo as frequências de $4 \mathrm{kHz}$ e $6 \mathrm{kHz}$ as mais afetadas, pois 48,1\% e 52,8\%, respctivamente, da amostra apresentaram redução dessa amplitude nessas frequências.

Diante dos resultados discutidos anteriormente, existem indicativos de que o uso das EOA possa servir de ferramenta auxiliar no diagnóstico precoce da Painpse contribuindo para, prevenção auditiva, uma vez que o estudo mostrou uma porcentagem de sujeitos que apresentam disfunção coclear (CCE) nas frequências de $4 \mathrm{kHz}$ e $5 \mathrm{kHz}$, mesmo sem terem alterações na audiometria tonal. Nesse sentido, destaca-se a importância da continuidade dos estudos nessas áreas, pois a compreensão dos efeitos do ruído elevado na fisiologia coclear torna-se fundamental para a implantação de programas de prevenção da saúde auditiva. Após aproximadamente 20 anos de implantação dos Programas de Conservação Auditiva no Brasil, acredita-se que o foco de atuação das equipes interdisciplinares nessa área deva passar a ser a prevenção e a qualidade de vida dos trabalhadores. Assim, a busca por ferramentas cada vez mais eficazes para o diagnóstico precoce e que possam contribuir significativamente para o planejamento e a aplicação de programas de promoção da Saúde Auditiva torna-se um diferencial importante para a efetividade desses programas.

\section{Conclusão}

Os resultados obtidos mostraram que as EOAT estiveram ausentes em maior número de respostas comparadas aos registros das EOAPD nesta amostra, mesmo quando os limiares auditivos obtidos na Audiometria Tonal Liminar estavam normais. Assim sendo, as EOAT são uma importante ferramenta para a detecção precoce de alterações na fisiologia coclear decorrente da exposição ocupacional ao ruído, permitindo que medidas preventivas e recuperativas sejam adotadas mais precocemente pelas empresas.

\section{Contribuições de autoria}

Ramos, N.: participou da elaboração do projeto, da coleta de dados, da análise, da tabulação dos dados, da interpretação dos resultados e da revisão da versão final. Aita, A. R. C.; Siqueira, L. P.; Aita, F. S.: participaram da elaboração do projeto, da coleta dos dados, da análise e interpretação dos resultados e da revisão da versão final. 


\section{Referências}

ALMEIDA, S. I. C. D. et al. História natural da perda auditiva ocupacional provocada por ruído. Revista da Associação Médica Brasileira, São Paulo, v. 46 n. 2, p. 143-158, 2000.

ATCHARIYASATHIAN, V.; CHAYARPHAM, S.; SAEKHOW, S. Evaluation of noise-induced hearing loss with audiometer and distortion product otoacustic emissions. Journal of the Medical Association of Thailand, v. 91, n. 7, p. 1066-1071, 2008.

BRASIL. Ministério do Trabalho e Emprego. Portaria $\mathrm{n}^{\circ}$ 19, de 9 de abril de 1998. Estabelece diretrizes e parâmetros mínimos para avaliação e acompanhamento da audição em trabalhadores expostos a níveis de pressão sonora elevados. Diário Oficial [da] República Federativa do Brasil, Poder Executivo, Brasília, DF, 22 abr. 1998. Seção I, p. 64-66. Disponível em: <portal.mte.gov.br/legislacao/ portaria-n-19-de-09-04-1998.htm > . Acesso em: 30 out. 2009.

COSTA, E. A. Classificação e quantificação das perdas auditivas e audiometrias industriais. Revista Brasileira de Saúde Ocupacional, São Paulo, v. 16, n. 61, p. 35-38, 1988.

FIORINI, A. C. O uso de registros de emissões otoacústicas como instrumento de vigilância epidemiológica de alterações auditivas em trabalhadores expostos a ruído. 2000. 160 f. Tese (Doutorado em Saúde Ambiental)-Faculdade de Saúde Pública, Universidade de São Paulo, São Paulo, 2000 .

GATTAZ, G. Contribuições do registro das emissões otoacústicas evocadas no diagnóstico das perdas auditivas induzidas pelo ruído. In: NUDELMANN, A. A. et al. PAIR - Perda Auditiva Induzida pelo Ruído. Rio de Janeiro: Revinter, 2001. v. 2.

JERGER, J. Clinical experience with impedance audiometry. Archives of Otolaryngoly, Chicago, v. 92, n. 4, p. 311-324, oct. 1970 .
KEMP, D. T. Otoacoustic emissions, their origin in cochlear function, and use. British Medical Bulletin, v. 63, p. 223-241, 2002.

KORRES, G. S. et al. Distortion product otoacoustic emissions in an industrial setting. Noise Health, v. 11, n. 43, p. 103-110, 2009.

MARQUES, F. P. Perda auditiva induzida por ruído: registro das emissões otoacústicas por produto de distorção como método de diagnóstico precoce. 2003. 105 f. Dissertação (Mestrado em Saúde Ambiental)Faculdade de Saúde Pública, Universidade de São Paulo, São Paulo, 2003.

MARQUES, F. P.; COSTA, E. A. Exposição ao ruído ocupacional: alterações no exame de emissões otoacústicas. Revista Brasileira de Otorrinolaringologia, São Paulo, v. 72, n. 3, p. 362-366, maio/jun. 2006.

MORAN, M. E. S. P.; FIORINI, A. C. Aplicações clínicas das emissões otoacústicas - produto de distorção em indivíduos com perda auditiva induzida por ruído ocupacional. Distúrbios da Comunicação, São Paulo, v. 14, n. 2, p. 237-261, 2003.

OLIVEIRA, T. M. T. Emissões otoacústicas em trabalhadores normo-ouvintes expostos ao ruído ocupacional. 1999. 88 f. Tese (Doutorado em Medicina)-Escola Paulista de Medicina, Universidade Federal de São Paulo, São Paulo, 1999.

SALAZAR, A. M. B. et al. Comparación de emisiones otoacústicas producto de distorsión en individuos expuestos y no expuestos a ruido ocupacional. Ciencia $\mathcal{E}$ Trabajo, Santiago, v. 5, n. 10 p. 24-32, 2003.

SLIWINSKA-KOWALSKA, M. The role of evoked and distortion-product otoacoustic emissions in diagnosis of occupational noise-induced hearing loss. Journal of Audiological Medicine, v. 7, n. 1, p. 29-45, 1998.

SOUSA, L. C. A. et al. Eletrofisiologia da audição e emissões otoacústicas: princípios e aplicações clínicas. São Paulo: Tecmedd, 2008. 